\title{
ICEF2020-2911
}

\section{Prediction of $\mathrm{NO}_{x}$ Emissions For a Range of Engine Hardware Configurations Using Artificial Neural Networks}

\author{
Nick Papaioannou, XiaoHang Fang, Felix Leach and Martin H. Davy \\ Thermal Propulsion Systems Research Group \\ Department of Engineering Science \\ University of Oxford \\ Oxford, OX1 3PJ, United Kingdom \\ Email: xiaohang.fang@eng.ox.ac.uk
}

\begin{abstract}
The predictive ability of artificial neural networks where a large number of experimental data are available, has been studied extensively. Studies have shown that ANN models are capable of accurately predicting $\mathrm{NO}_{x}$ emissions from engines under various operating conditions and different fuel types when trained well. One of the major advantages of an ANN model is its ability to relearn when new experimental data is available, thus continuously improving its accuracy. The present work explored the potential of an ANN model to predict $\mathrm{NO}_{x}$ emissions for various engine configurations outside its training envelop. This work also looked into quantifying the amount of new data required to improve the accuracy of the model when exposed to unknown conditions. The chosen ANN model was constructed using data from a high-speed direct injection diesel engine and is capable of accurate $\mathrm{NO}_{x}$ emissions over a wide range of operating conditions. The optimized network utilized 14 input parameters and is using 6 neurons in a single hidden layer feed-forward neural network. Experimental data from the various engine configurations tested, were then used to predict $N O_{x}$ from the existing ANN model. The results indicate that when the new data are within the baseline training envelop, the ANN model is capable of accurate $N O_{x}$ prediction even when there are substantial changes in engine configuration such as piston material. Similar results were also observed when the injector nozzle is changed. However, the model's performance drops significantly when new data, outside the baseline training envelop, were employed in-
\end{abstract}

dicating that additional training is required. As such, various methods for retraining the ANN model were explored with the selected method showing the best compromise between new-data accuracy and old-data accuracy retention. The retrained ANN model developed was found to be an effective tool in predicting $\mathrm{NO}_{x}$ emissions for different engine configurations and operating conditions.

$\begin{array}{ll}\text { NOMENCLATURE } \\ \text { AFR } & \text { Air Fuel Ratio } \\ \text { ANN } & \text { Artificial Neural Network } \\ \text { CAD } & \text { Crank Angle Degree } \\ \text { COV } & \text { Coefficient of Variation } \\ \text { EGR } & \text { Exhaust Gas Recirculation } \\ \text { ICE } & \text { Internal Combustion Engines } \\ \text { gIMEP } & \text { Gross Indicated Mean Effective Pressure } \\ \text { nIMEP } & \text { Net Indicated Mean Effective Pressure } \\ \text { nISFC } & \text { Net Indicated Fuel Consumption } \\ \text { LM } & \text { Levenberg-Marquardt } \\ \text { LTC } & \text { Low Temperature Combustion } \\ \text { mfb 50\% } & \text { Point of 50\% mass-fraction burned } \\ \text { mfb 90\% } & \text { Point of 90\% mass-fraction burned } \\ \text { MLP } & \text { Multi-layer Perceptron } \\ \text { MSE } & \text { Mean Square Error } \\ \text { ROHR } & \text { Rate of Heat Release } \\ r & \text { Pearson correlation } \\ R^{2} & \text { Coefficient of determination }\end{array}$




\section{INTRODUCTION}

Diesel engines are known for their reliability, high thermal efficiency and high-torque output in various industries including ground transportation, heavy-duty machinery and stationary power generation. In order to benefit from these advantages, modern diesel engine designs need to meet stringent emission regulations [1]. As such, low $\mathrm{NO}_{x}$ emissions are an essential design specification during the development stage, which should be met without compromising thermal efficiency. Various incylinder solutions have been developed and used in modern engines which are primarily designed to enhance fuel-air mixing under highly-diluted conditions [2-4]. Advancing the main injection into the intake stroke or increasing exhaust gas recirculation (EGR) to high levels, can suppress in-cylinder peak flame temperature, resulting in a simultaneous reduction of $\mathrm{NO}_{x}$ and soot emissions - so-called low temperature combustion (LTC). In-cylinder swirl has also been found to be an effective way of enhancing fuel-air mixing, thus reducing soot emissions and in some cases enhancing combustion efficiency [5,6]. Equally, the use of multiple injections during a single cycle is also a well accepted method of improving emissions and performance [7,8].

Automotive manufacturers most commonly rely on rigorous experimental test schedules over a wide range of operating conditions in order to validate and adopt promising emission-reduction strategies. Various numerical methods have been proposed to shorten this strenuous process in evaluating engine performance and emissions characteristics. Researchers have used design of experiments, multivariate analysis, linear correlations, and some symbolic regression algorithms to validate design configurations $[9,10]$. However, the stochastic, complex and nonlinear nature of the emissions' formation posed a significant challenge on traditional models where accurate prior knowledge of the physical process is needed.

In recent years, emerging artificial intelligence technologies and algorithms have shown great potential in predictive studies for a wide range of scientific fields involving nonlinear processes. In the field of engine research, studies have favoured Artificial Neural Networks (ANN) as a predictive modelling and design tool [11-17]. Compared to other predictive non-linear models, the main advantage of ANN lies in its ability to identify cryptic, nonlinear, highly complex correlations, between the measured input and output data. Some of the important features that have contributed to their popularity include their ease of implementation, their ability to learn from examples without explicit knowledge of the underlying physics, and their powerful generalisation abilities. Being a machine learning tool, an ANN also has the ability to relearn when new data is available, which can further increase the model's accuracy. Additional input and output variables can also be added/removed if necessary.

Various researchers have studied the application of an ANN in combustion characterization, including the optimization of reaction rate parameters in chemical kinetic modelling [18] and combustion control [19]. A number of studies have focused on predicting the emission and combustion characteristics of internal combustion engines (ICEs) by using the ANN approach. Parlak et al., [20] examined the feasibility of using ANN to predict specific fuel consumption and exhaust temperature for a diesel engine. Mauro et al., [21] investigated the ability of neural networks to accurately model the indicated mean effective pressure (IMEP) and its coefficient of variation (COV of IMEP) in a spark-ignited internal combustion engine. ANN models have been also successfully applied to a wide range of engine design problems including diesel engine controller design [22] and mechatronic control system design [23].

Recently the authors of this study used an ANN model to accurately predict the $\mathrm{NO}_{x}$ emissions of a high-speed direct injection diesel engine. The study focused on identifying the most important engine parameters for accurate $\mathrm{NO}_{x}$ prediction over a wide range of operation. This study highlighted 14 parameters that have the biggest effect on $\mathrm{NO}_{x}$, based on a Pearson correlation analysis, and shown that ANN models are capable of accurately predicting $\mathrm{NO}_{x}$ emissions even when tested outside the training range of the model [24]

Despite their powerful generalisation abilities, like other empirical models, their performance deteriorates when deployed outside their training range. By using the widest limits of the data during training provides the best chance of extrapolating for an ANN model. However, once a trained ANN model has been deployed in an operational setting, it is still likely to perform poorly if faced with new input patterns that are far removed from its training range. Therefore, when new data is available, it is important to seek to improve the extrapolation ability.

This can be achieved either through improved learning algorithms, catering real-time predictions or updating the existing ANN model parameters (i.e. retrain the model) in real-time during deployment. To the best of the authors' knowledge, limited or no study has been performed to study the ability of an ANN model in predicting $\mathrm{NO}_{x}$ emissions outside its training range. Therefore, in this work, we have used an existing ANN model as a design tool in predicting $\mathrm{NO}_{x}$ emissions for various engine hardware configurations. A focus was given in determining the number of new test-points required to retrain the model and achieve acceptable accuracy, for a given engine configuration, while accurately predicting $\mathrm{NO}_{x}$ emissions outside the training range of the model.

\section{EXPERIMENTAL APPARATUS}

The data used in this work are results of engine testing on a single-cylinder, high-speed direct injection diesel engine. A detailed description of the engine design and instrumentation is presented in previous work [25]. The data were logged at a frequency of $1 \mathrm{~Hz}$ using a Sierra-CP Cadet system and each testpoint was logged for $180 \mathrm{~s}$ and several repeats were carried out 
TABLE 1: Baseline engine parameters

\begin{aligned} & \hline Parameters Specifications \\ & \hline Bore x Stroke $83 \mathrm{~mm}$ x $92.4 \mathrm{~mm} \\ &$ Displacement $500 \mathrm{~cm}^{3} \\ &$ Valves per cylinder 2 intake, 2 exhaust \\ & Fuel system Common rail \\ & Fuel injector 8-hole solenoid \\ & \hline\end{aligned}

for statistical analysis. Table 1 presents the details of the test engine.

For this work data from various engine hardware configurations were used to assess the applicability of an existing ANN model, trained on a completely different hardware setup, in predicting $\mathrm{NO}_{x}$ emissions. More specifically, different piston materials, cylinder heads, injector types, nozzle types and injection strategies were all tested using a combination of the above parameters. The experimental findings for these configurations are detailed in literature [26,27]. Table 2 shows the engine configurations that were tested in this work, along with the baseline configuration.

It is worth noting that the piston bowl design was the same between the different configurations, as was the compression ratio, and only the piston material was altered. Configuration 1, used an aluminium piston which employed an active thermal coating as a method of improving thermal efficiency [27]. This coating allows the piston surface temperature to better follow the instantaneous in-cylinder temperature, due to a combination of low heat capacity and thermal conductivity, thus reducing the heat transferred to the piston walls. Furthermore, two different injector technologies were tested, solenoid and piezoelectric. Due to the faster response of the piezoelectric injectors, different injection strategies were employed, compared to the baseline configuration (i.e. 5 injections per cycle vs 3 injections per cycle for the solenoid injectors).In addition, different injector nozzles were tested, which varied in the number of holes, cone spray angle and $k$-factor values. Finally, two cylinder head types were tested, which due to their inherent design characteristics allowed for different levels of in-cylinder swirl.

Table 3 shows the test-points used for each engine configuration. It should be noted that test-points (TP) 1 and 2 were used for the baseline configuration and engine Config. 1-2, whereas TP3-4 were used for Config. 3. The reasons for this were the different requirements for the different injector technologies as well as the lower in-cylinder swirl resulting from the different cylinder head design. All test-points were run over a 3, 5 or a 7point EGR sweep depending on the test requirements and, where available, the error bars indicate one standard deviation over an average of three runs. It should be noted that all emissions data have been rescaled by dividing the values by an arbitrary number (the same value for every plot) for reasons of commercial confidentiality.

\section{NUMERICAL METHODOLOGY}

In this study, a commonly recognized and used ANN structure, multilayer perceptron (MLP, sometimes loosely referred to as the feedforward ANN), was constructed. MLP uses a supervised learning method called backpropagation to train the neural network model. A typical structure for this type of ANN can be seen in Figure 1. It consists of one input layer, a number of hidden layers and one output layer. With the exception of the nodes in the input layer, each node is known as a neuron.

An activation function is needed in the hidden layer and the output layer in order to introduce non-linearity in the model. A widely used activation function is the continuous differentiable log-sigmoid function given by:

$$
h(x)=\frac{1}{1+e^{-x}}
$$

The neural network function can be written as:

$$
y_{k}(\mathbf{x}, \mathbf{w})=\sigma\left(\sum_{j=1}^{N} w_{k j}^{(2)} h\left(\sum_{i=1}^{M} w_{j i}^{(1)} x_{i}+w_{j 0}^{(1)}\right)+w_{k 0}^{(2)}\right)
$$

where parameters $w_{j k}^{(1)}$ are the weights and parameters $w_{k 0}^{(2)}$ are biases. Vector $\mathbf{w}$ represents the grouped weights and biases in the system. In order to find the optimal value $\mathbf{w}$ for the model, we need to minimize the error of the network. The error function used here is the mean squared error (MSE) function given by:

$$
\left.E(\mathbf{w})=M S E=\frac{1}{N} \sum_{i=1}^{N}\left(y_{(} x_{n}, \mathbf{w}\right)-t_{n}\right)^{2}
$$

where $t_{n}$ is the target output. There are different ways to minimize the error function. For a feed-forward neural network structure, back propagation learning algorithms are widely used.

The authors have previously studied the effect of different backpropagating learning algorithms where the LevenbergMarquardt (LM) [9] algorithm is found to be most computationally efficient for the amount of data that is used. LM is a secondorder method providing faster and more accurate solutions owing to its inclusion of second-order derivative of error information. The LM algorithm has been successfully used for training in engineering applications with significant advantages over other methods when given a median-sized dataset [13], [22]. In order 
TABLE 2: Engine configurations used in this work

\begin{tabular}{|c|c|c|c|c|}
\hline & Baseline config. & Config. 1 & Config. 2 & Config. 3 \\
\hline Piston material & Aluminium & Al-thermally coated & Steel & Steel \\
Injector type & Solenoid & Solenoid & Solenoid & Piezo \\
Cylinder head & hi-swirl & hi-swirl & hi-swirl & low-swirl \\
Injections per cycle & 3 & 3 & 3 & 5 \\
\hline
\end{tabular}

TABLE 3: Engine operating conditions for the test-points presented in this work

\begin{tabular}{|c|c|c|c|c|}
\hline Test-point (TP) & $\mathbf{1}$ & $\mathbf{2}$ & $\mathbf{3}$ & $\mathbf{4}$ \\
\hline Speed (RPM) & 1500 & 2000 & 1500 & 2000 \\
nIMEP (bar) & 6.9 & 25.7 & 3.8 & 12.3 \\
Rail pressure (MPa) & 67 & 153 & 73 & 164 \\
Boost pressure (barG) & 0.46 & 1.9 & 0.04 & 0.76 \\
Back-pressure (barG) & 0.84 & 2.9 & 0.3 & 1.0 \\
Inlet Temperature $\left({ }^{\circ} \mathrm{C}\right)$ & 40 & 40 & 55 & 62 \\
EGR $(\%)$ & $0-40$ & $0-12$ & $0-60$ & $0-25$ \\
\hline
\end{tabular}

to avoid over fitting while training the network, the data is split into three parts for training, validation and testing. The training and testing datasets should include representative samples of all the cases the network handles. For this work the data were split as follows; training: $70 \%$, validation: $10 \%$, and testing: $20 \%$ and the data selection for each sub-set was carried out randomly to remove any training bias. More importantly, complete independent verification datasets are also set aside for further testing to ensure the generalisation of the ANN model when new data are introduced.

A detailed study on the model's controlling parameters reviewed that out of the original 41 parameters (which were standard engine test bed logged parameters), 14 parameters were shown to be critical in modelling the effects of different engine operating conditions on $\mathrm{NO}_{x}$ prediction. These parameters are detailed in Table 4. A sensitivity test was also performed to determine the optimum number of neurons in the hidden layer. Following the results of the sensitivity test, the ANN consists of one hidden layer with 14 input nodes, 6 neurons in the hidden layer and 1 output neuron. The aforementioned model and algorithms were implemented in MATLAB.

\section{Retraining methodology}

The retrain methodologies can be generally divided into two main categories: online and offline. Online retraining entails when a new observation is obtained; the observation is used to further train the model. The current model is further trained

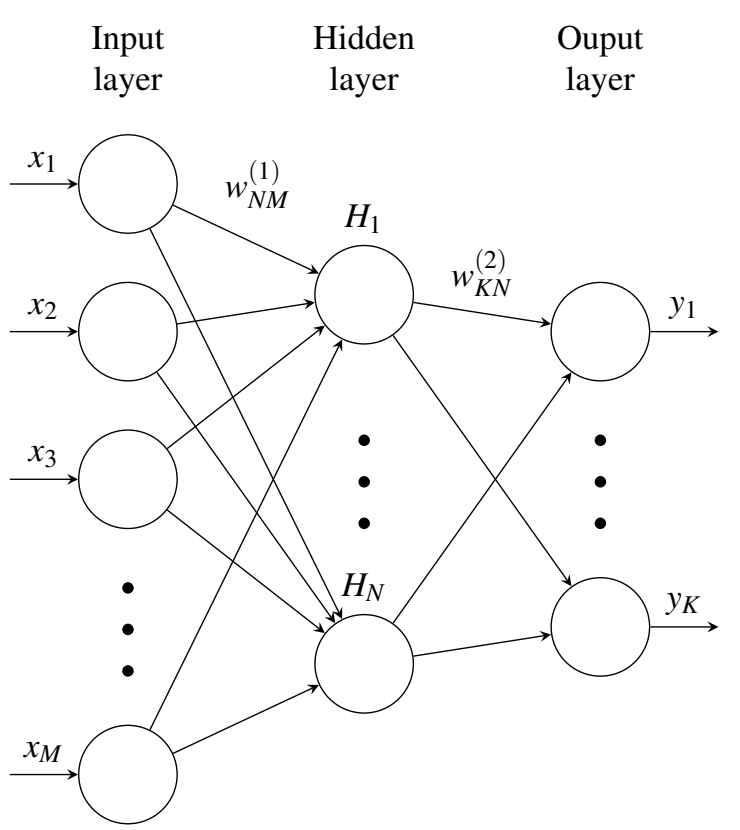

FIGURE 1: Schematic diagram of a feedforward neural network

by backpropagating via the single observation. This approach "learns" in a sequential manner where the model adapts locally to the data in that it will be more influenced by the recent observations than by older observations. This approach is suitable when the model needs to dynamically adapt to new patterns in the data or when an extremely large dataset is given, which makes it impossible to retrain for the whole set.

Offline retraining also occurs when a new observation is obtained however the single data point is appended to the existing training set. The retraining is then performed on the new, appended dataset. This approach leads to a better global approximation of the target function and is suitable when a fixed new dataset is given. In this study, the size of the new dataset is considerably less than the baseline data; therefore, offline retraining was performed. A complete retrain algorithm is given in Figure 2. 
TABLE 4: Engine parameters in order of importance based on the Pearson correlation coefficient

\begin{tabular}{lc}
\hline Parameters & Pearson correlation \\
\hline EGR & -0.8158 \\
Volumetric efficiency & 0.7737 \\
Inlet mass flow rate & 0.5384 \\
Exhaust mass flow rate & 0.5356 \\
Inlet temperature & -0.4843 \\
EGR cooler outlet $\mathrm{T}$ & -0.4537 \\
Cylinder $\mathrm{P}_{\text {max }}$ & 0.4513 \\
gIMEP & 0.4360 \\
Coolant jacket $\delta \mathrm{T}$ & 0.4095 \\
Brake power & 0.4059 \\
Net power & 0.4034 \\
Cylinder head coolant $\delta \mathrm{T}$ & 0.3988 \\
Inlet Pressure & 0.3892 \\
Fuel flow rate & 0.3838 \\
\hline
\end{tabular}

\section{RESULTS}

Initially, the training procedure and accuracy of the baseline ANN model is detailed. Then, the different engine configurations (Table 1) were tested with the baseline ANN model and an assessment on its suitability to predict $\mathrm{NO}_{x}$ emissions under different engine configurations was made. A success threshold was set to \pm 50 arbitrary units of $\mathrm{NO}_{x}$ - which corresponds to $3 \%$ of full scale accuracy of the emissions analyser. If the model error was outside the aforementioned threshold, then experimental data from that engine configuration were used to retrain the baseline ANN model. The retraining process was continued until the success threshold was met. The results of this process are shown in the second half of this section. The global model's accuracy (i.e. model accuracy after retraining) was then tested using a series of independent test conditions for each engine configuration. Finally, an estimation on the required engine testing time needed to $\log$ the necessary data for retraining each engine configuration was also made.

\section{Baseline ANN model}

The baseline ANN model was trained using data taken with the baseline engine configuration shown in Table 2. The training data covered a wide range of engine speed and load conditions as shown in Figure 3. For each speed and load condition a 5-point EGR sweep was performed and several repeats were logged depending on the testing objectives. Each point on the EGR sweep was logged for $180 \mathrm{~s}$, as already mentioned in previous sections. This resulted in a total of 128,000 training points for the ANN model. The training results showed a high model accuracy with the $R^{2}$ values for training, validation and testing being 0.994 , 0.992 and 0.994 respectively. Finally, the predictive ability of the model was tested on two verification test-points that were

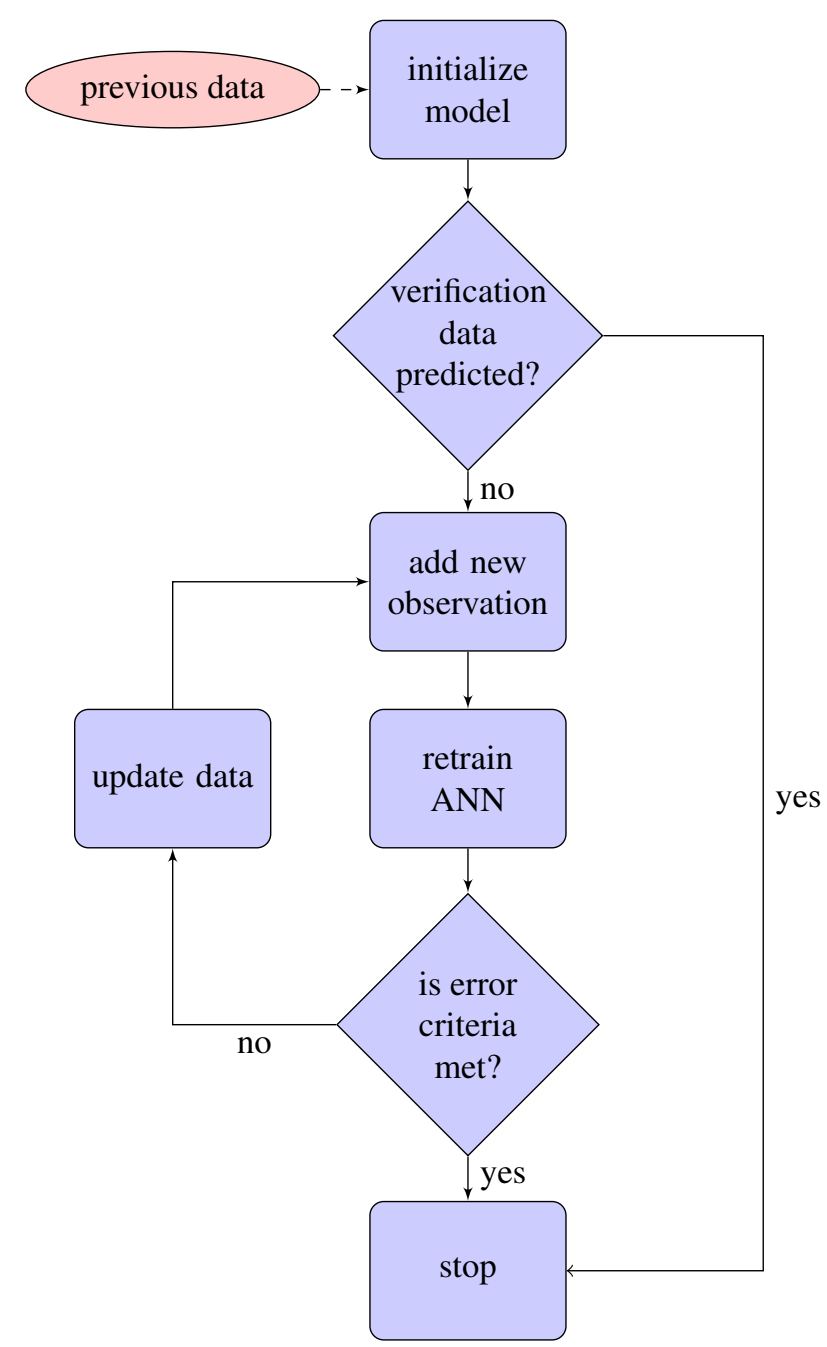

FIGURE 2: Conceptual approach to model retrain

completely independent from the training data. This ensured that the model can generalise well outside its training range. These points are shown in orange in Figure 3.

Figure 4 shows the baseline model results for the verification conditions highlighted in Figure 3. As it can be seen, a very good agreement between the ANN model and the experimental data exists throughout the whole EGR range. These results indicate that we can confidently predict $\mathrm{NO}_{x}$ emissions (the error is $<100$ arbitrary $\mathrm{NO}_{x}$ units across the whole range) even for conditions that the ANN model has not been trained for. The following section, will present the use of this model (henceforth referred to as the baseline model) and its ability to predict $\mathrm{NO}_{x}$ emissions for a variety of engine configurations, that has not been trained for (refer to Table 2). 


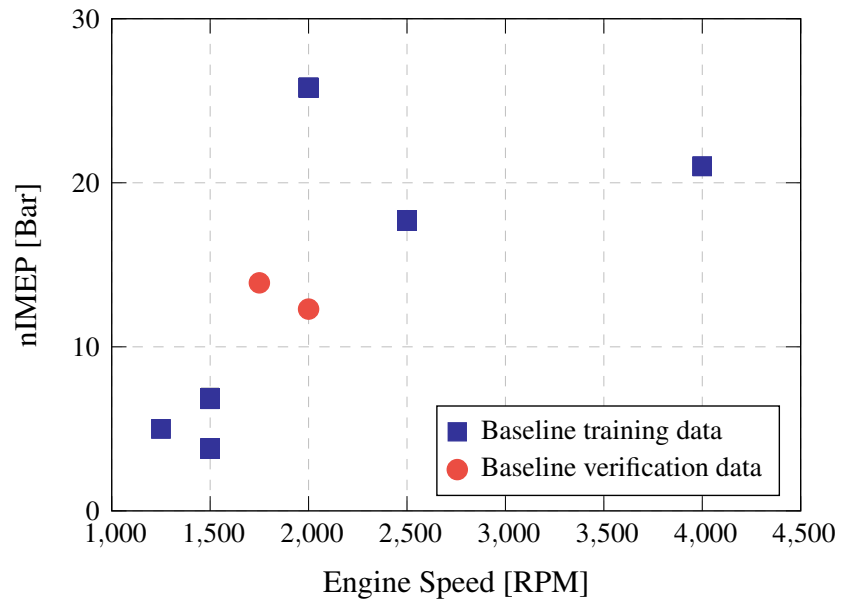

FIGURE 3: Baseline ANN training envelop in terms of engine speed and load. The training data for the ANN model are shown in blue and the verification data in orange.

\section{Testing the baseline ANN model with new data from different engine configurations}

Figure 5 shows the absolute error in $\mathrm{NO}_{x}$ prediction under TP1 and 2 conditions for Configuration 1. The results show that the baseline ANN model can predict $\mathrm{NO}_{x}$ very accurately for both conditions, with the biggest error no larger than \pm 40 arbitrary $\mathrm{NO}_{x}$ units when the error bars are taken into consideration. It is also worth noting the small size of the error bars $(<20$ arbitrary $\mathrm{NO}_{x}$ units), which highlights the accuracy of the experimental data as well as the good repeatability of the ANN model. These results are in agreement with the emissions' performance of the thermally coated piston where very small differences were observed in terms of $\mathrm{NO}_{x}$ when compared to the baseline engine configuration [27]. Furthermore, the 14 input parameters to the ANN model for Configuration 1, showed very small deviation from the baseline configuration, implying that the model is exposed to conditions that are already trained for, thus resulting in the small errors observed.

Equally good accuracy is observed for Configuration 2 under TP1 conditions, with the error increasing to a maximum of \pm 80 arbitrary $\mathrm{NO}_{x}$ units at $0 \% \mathrm{EGR}$ rate (Figure 6). This again indicates that the baseline model can accurately predict $\mathrm{NO}_{x}$ emissions under these operating conditions even when the piston material is different. However, a completely different picture can be seen as the speed and load increases. Here the error is significant when the baseline ANN model is used, across the whole EGR sweep, with the highest error value observed at 0\% EGR. Considering that the input parameters for these conditions where within the training range of the baseline model, it seems that the model cannot capture some of the underlying physics associated with Configuration 2 (notably the use of steel pistons) and result-
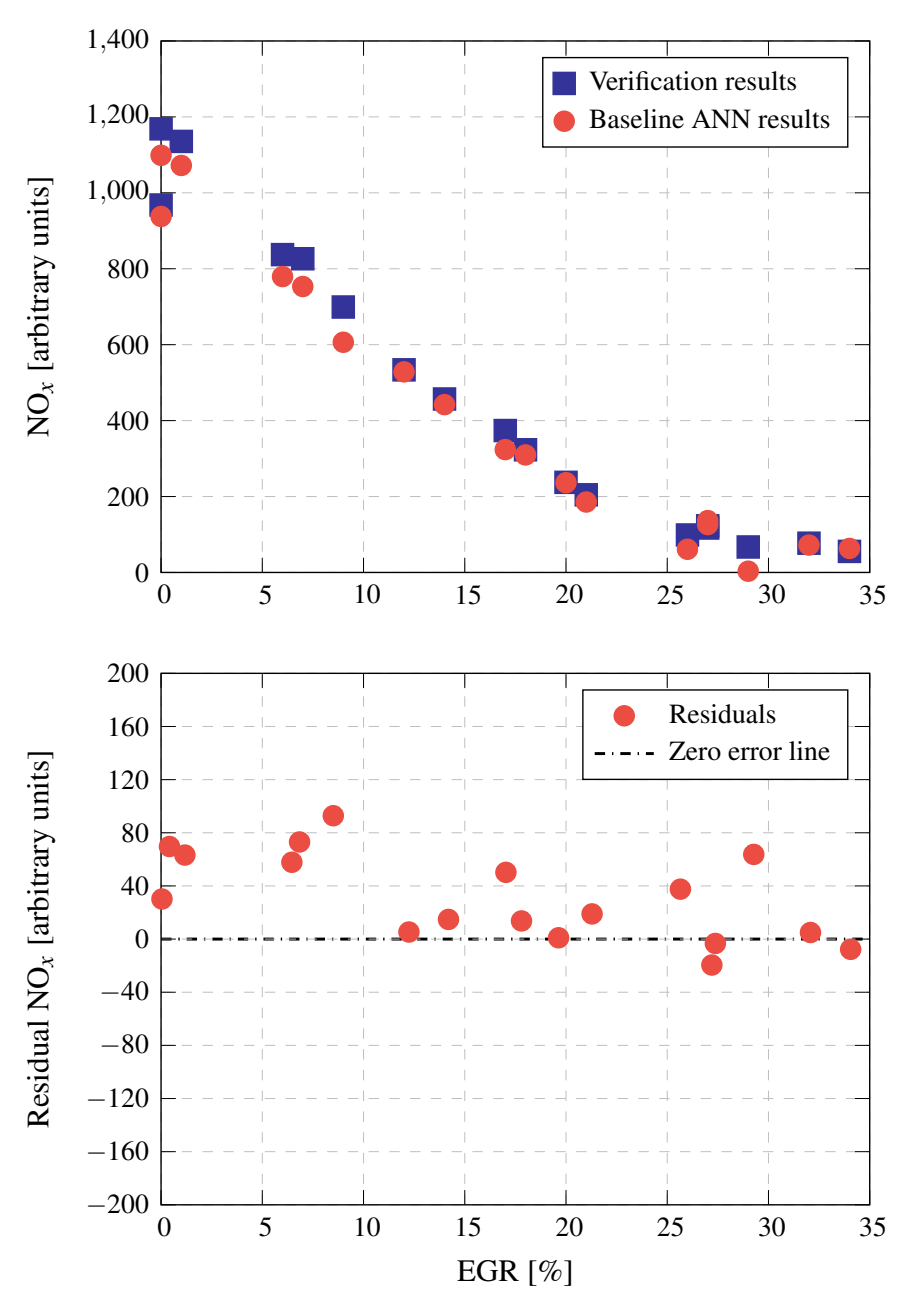

FIGURE 4: Baseline ANN model results for the verification data shown in Figure 3 (top) and absolute error across the whole range (bottom)

ing $\mathrm{NO}_{x}$ emissions.

The use of steel pistons is a relatively new solution to improve thermal efficiency in the light-duty diesel sector. Due to the lower thermal conductivity of steel (compared to aluminium, which is the most common piston material used), heat transfer is reduced, resulting in higher peak cylinder temperatures and pressures thus increasing the potential work output per cycle [28]. Studies also suggest that this increase in peak cylinder temperature has the potential to increase burn rates and improving combustion efficiency [29], but with a potential increase in thermal $\mathrm{NO}_{x}$ - unless $\mathrm{NO}_{x}$ emissions are kept constant during testing [30]. This would imply that all things being equal, if the resulting $\mathrm{NO}_{x}$ values are expected to be higher for the steel piston, then the baseline ANN model would under-predict the target $\mathrm{NO}_{x}$ values, which is what we observe in Figure 6. As such, the ANN model 
will have to be retrained for the data of Configuration 2 in order to capture the effects of steel piston on $\mathrm{NO}_{x}$ emissions.

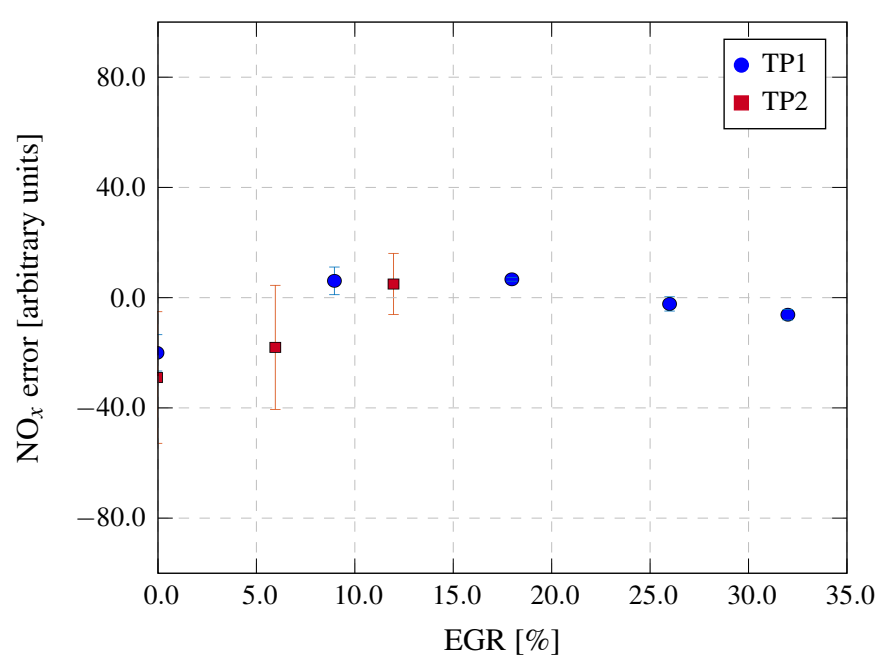

FIGURE 5: $\mathrm{NO}_{x}$ predictions with baseline ANN model at TP1 and TP2 conditions for Engine Config. 1. The error bars indicate one standard deviation over an average of three runs.

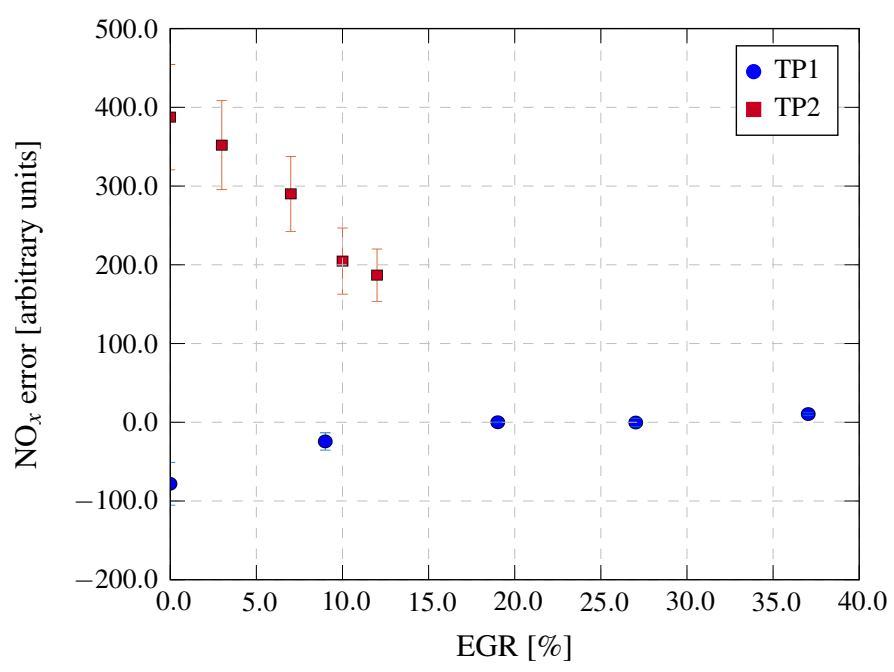

FIGURE 6: $\mathrm{NO}_{x}$ predictions with baseline ANN model at TP1 and TP2 conditions for Engine Config. 2. The error bars indicate one standard deviation over an average of three runs.

Figure 7 shows the error in $\mathrm{NO}_{x}$ prediction for Configuration 3 under TP3 and TP4 conditions when the baseline ANN model is used. In this instance, a single seven-point EGR sweep was performed for each condition (no repeat tests), and consequently, the use of error bars is not possible. Figure 7 shows relatively small errors that increase slightly as the EGR rate is reduced. On the other hand, under TP4 conditions, the error is significantly higher across the whole range, increasing significantly as EGR is reduced. This difference in results between the two speed/load conditions is quite interesting, considering the significant hardware changes from the baseline configuration with Configuration 3 (primarily the change of fuel injector technology from solenoid to piezoelectric, and a number of different fuel injectors tested as well as a reduction in swirl ratio).

The use of piezoelectric injectors allows for a much faster response, compared to the solenoid system, thus increasing the flexibility in the number of injections used during the cycle. This allows more injections during any one time, which improves fuel-air mixing as the fuel quantity injected is reduced. Following that, the injection strategy used with the piezoelectric injectors was significantly different from the baseline configuration both in terms of the number of injections as well as injection separation. This is expected to change the mixing conditions in the cylinder. Finally, reducing the in-cylinder swirl is also expected to reduce fuel-air mixing caused by swirl (although these effects could be compensated by an increased number of injections), thus affecting the emission formation process.

These results then indicate that despite the differences in engine hardware and injection strategy, the expected changes in fuel-air mixing, have a minimal effect on $\mathrm{NO}_{x}$ formation under low speed and load, thus the baseline ANN model can be used without retraining. On the other hand, as speed and load increases, less time is available for mixing, and it is expected that the fuel-air mixing process will begin to dominate emission formation. Consequently, a model trained to capture such effects for the baseline configuration cannot be used if the fuel-air mixing process is significantly different.

Based on these findings, a retraining procedure is necessary for Configurations 2-3, in order to improve the model's accuracy under these hardware specific changes. The next section presents the results of this retraining procedure.

\section{ANN model retraining}

As already mentioned, the retraining process can be carried out either online or offline. It is therefore of practical importance to have an indication on the amount of new data required to retrain the ANN model, given an error range, and as such the required engine testing time needed to log these data. The retraining process in this study was carried out for every point of the EGR sweep until the predicted error was within the \pm 50 arbitrary $\mathrm{NO}_{x}$ units error constraint. Table 6 shows an estimate of how much engine testing time is required for every retraining process carried out here. It is recalled that the test conditions used for retraining are shown in Table 3. 


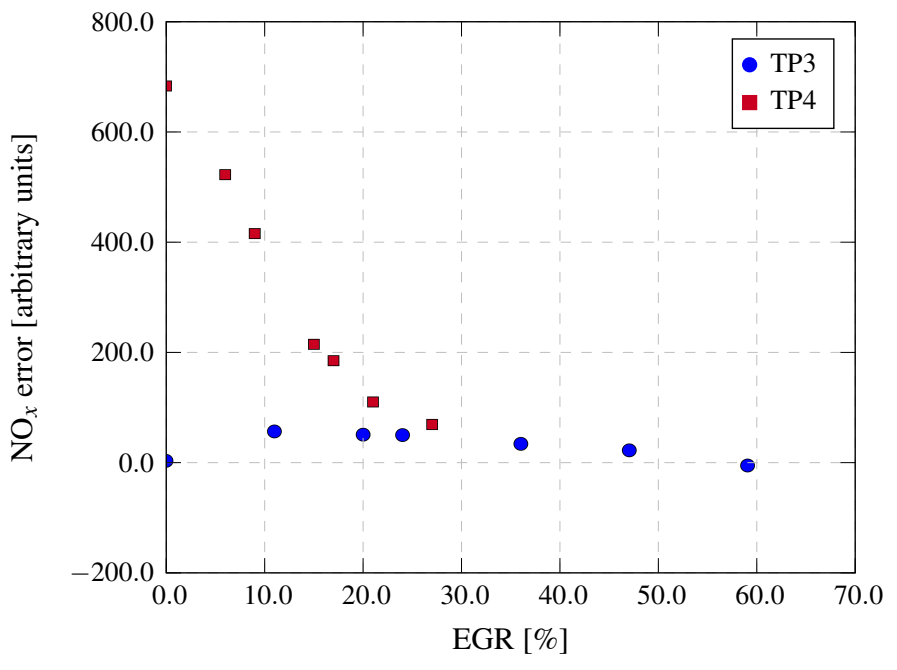

FIGURE 7: $\mathrm{NO}_{x}$ predictions with existing ANN model at TP3 and TP4 conditions for Engine Config. 3

TABLE 5: Engine operating conditions for the verification points used for model retraining under the various engine configurations

\begin{tabular}{|c|c|c|c|c|c|}
\hline Engine config. & \multicolumn{2}{|c|}{ Baseline } & \multicolumn{2}{|c|}{$\mathbf{2}$} & $\mathbf{3}$ \\
\hline Verification point (VP) & 1 & 2 & 3 & 4 & 5 \\
\hline Speed (RPM) & 1750 & 2000 & 2000 & 1500 & 1500 \\
nIMEP (bar) & 13.9 & 12.3 & 25.7 & 6.9 & 6.9 \\
Rail pressure (MPa) & 125 & 136 & 153 & 77 & 94.5 \\
Boost pressure (barG) & 1.1 & 1.2 & 2.1 & 0.46 & 0.295 \\
Back-pressure (barG) & 1.9 & 2.2 & 3 & 0.85 & 0.51 \\
Inlet Temperature ( $\left.{ }^{\circ} \mathrm{C}\right)$ & 40 & 40 & 40 & 40 & 63 \\
EGR (\%) & $0-30$ & $0-30$ & $0-20$ & $0-46$ & $0-40$ \\
\hline
\end{tabular}

The results shown in Table 6 indicate the number of additional $1 \mathrm{~s}$ test $\log$ s required on the engine, to meet the error criteria used in this work. It should be noted however, that the model's training time is not taken into account as this will be varying widely, depending on the training procedure (i.e. online or offline), CPU performance and algorithmic efficiency. Just as an indication, an upper bound in the model's training time was $\tilde{1} \mathrm{hr}$ of CPU time given a 4-core processor. Equally, the time required to rebuild the engine into a different hardware configurations is not taken into account.

Perhaps not surprisingly, TP2 and TP4 require the longest testing time, in agreement with the increased error values shown in Figures 6 and 7. Furthermore, only a few seconds of new test data are necessary to achieve the target accuracy for the low speed/load points (i.e. TP1 and TP3) where very small errors were observed. In total, for the conditions and training approach tested here, $\sim 15$ minutes of extra engine time is required to meet the error criteria selected.

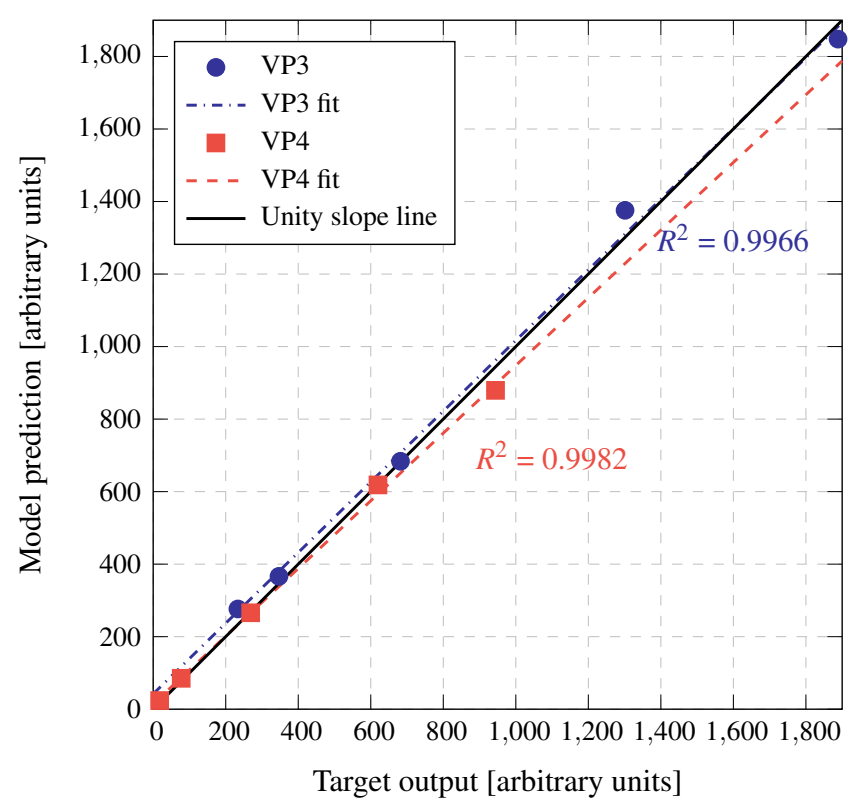

FIGURE 8: Verification results for Configuration 2 under conditions VP3 and VP4.

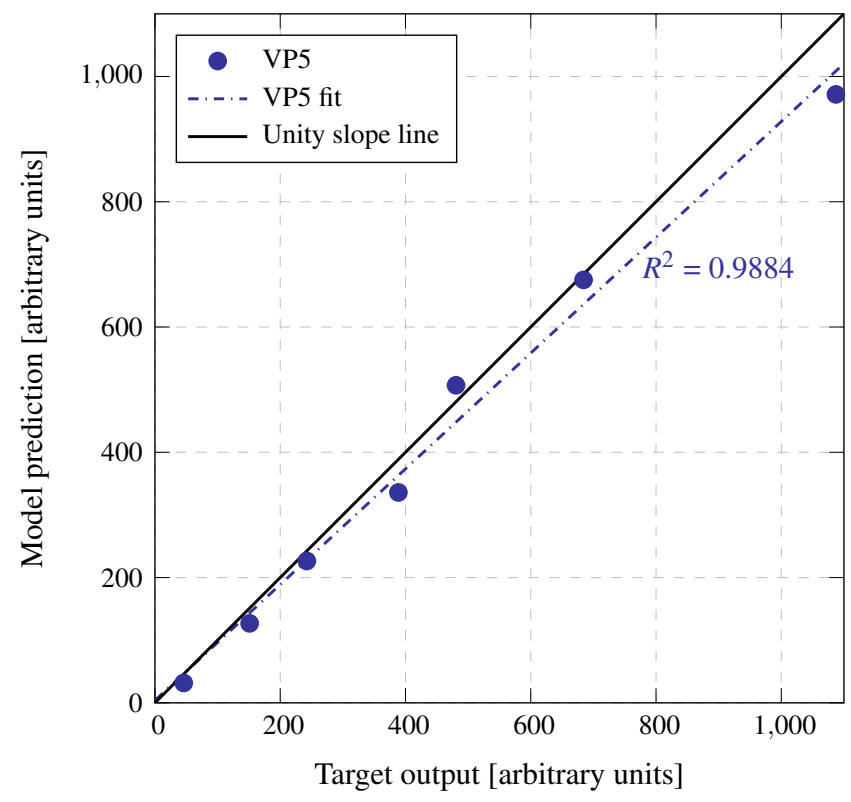

FIGURE 9: Verification results for Configuration 3 under conditions VP5.

It is recalled that, the goal of the retraining process is to improve the accuracy of the model for new cases without compromising the accuracy of the baseline predictions. The accuracy of the retrained model, as well as its generalisation in new cases (verification points (VP)), was tested on independent datasets after the model was retrained for the conditions shown in Table 3. The independent dataset for each engine configuration can be 
TABLE 6: Required engine test-time for retraining the ANN model under different engine configurations

\begin{tabular}{|c|c|c|c|c|}
\hline & \multicolumn{2}{|c|}{ Config. 2 } & \multicolumn{2}{c|}{ Config. 3 } \\
\hline Test-point & TP1 & TP2 & TP3 & TP4 \\
\hline Test time required (s) & 18 & 258 & 7 & 627 \\
\hline
\end{tabular}

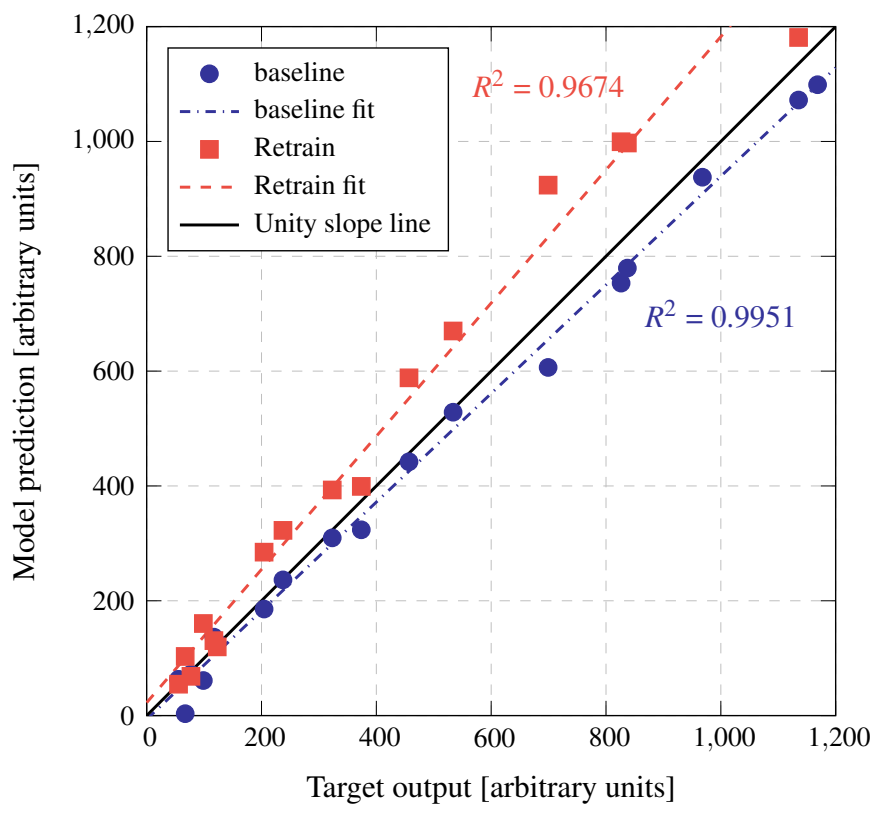

FIGURE 10: Verification results for the baseline configuration before and after the retraining process.

\section{seen in Table 5.}

Figure 8 shows the verification results for Configuration 2 . As can be seen, the model can now capture the effects of steel piston on $\mathrm{NO}_{x}$ and results in very good accuracy, even for data that have not been used for training. Equally good accuracy can be seen with Configuration 3, as shown in Figure 9. Finally, the accuracy of the baseline model, before retraining, can be seen in Figure 10 where the circles indicate the results before and the squares the results after retraining for a new engine configuration. More specifically, Figure 10 is showing the "loss" of accuracy for the baseline case when new engine configuration data are used to train the model. The retraining has caused some degradation on accuracy under these conditions and more specifically, under the high- $\mathrm{NO}_{x}$ region, causing the results to depart from the unity slop line. Most importantly, though, the low- $\mathrm{NO}_{x}$ region, which is inherently harder to predict and measure, shows almost no change between the two cases. These results then show that retraining can indeed result in accurate $\mathrm{NO}_{x}$ predictions for two completely different engine configurations without compromising its baseline performance.

\section{CONCLUSIONS}

This work has shown the utility of artificial neural networks in predicting $\mathrm{NO}_{x}$ emissions for a high-speed direct injection diesel engine under various hardware configurations. A baseline ANN model was developed and trained over a wide range of speed and load conditions. The generalisation of this model was tested using completely independent test-points. The results showed a small error ( $<100$ arbitrary $\mathrm{NO}_{x}$ units) when compared to the experimental data across the whole EGR range.

The baseline model was then used onto different engine configurations to test its predictive performance. The various configurations involved the use of different piston material and active thermal coating, injector type, and cylinder head. The results have shown that the baseline ANN model is capable of predicting $\mathrm{NO}_{x}$ emissions under low speed/load conditions independent of engine hardware configuration, for the conditions tested here. One the other hand, at higher speeds and loads the prediction error significantly increased. This is perhaps due to the fact that the $\mathrm{NO}_{x}$ emission response is more sensitive at these conditions as heat transfer and fuel/air mixing, become more dominant.

The baseline ANN model was retrained using new training data for each engine configuration to improve its performance at high speed and load conditions. Given the existing network configuration, it was estimated that in total 15 minutes of engine testing would be required to meet the error criteria selected. The final model's accuracy was tested using various independent test cases which ensured good predictive performance for the new engine configurations while retaining the accuracy for the baseline configuration. The results show a very good agreement in $\mathrm{NO}_{x}$ prediction for the new hardware configurations with only a slightly increase in error for the baseline configuration at high $\mathrm{NO}_{x}$ values. The accuracy at the low- $\mathrm{NO}_{x}$ range was retained.

\section{ACKNOWLEDGEMENT}

The authors would like to thank Jaguar Land Rover Limited, EPSRC, The China Oxford Scholarship Fund and The Great Britain-China Scholarship Fund for financial support.

\section{REFERENCES}

[1] Senecal, P., and Leach, F., 2019. "Diversity in transportation: Why a mix of propulsion technologies is the way 
forward for the future fleet". Results in Engineering, 4, p. 100060.

[2] Hasegawa, R., and Yanagihara, H., 2003. "HCCI Combustion in DI Diesel Engine". SAE Technical Paper, 2003-010745, 03,

[3] Kimura, S., Aoki, O., Ogawa, H., Muranaka, S., and Enomoto, Y., 1999. "New Combustion Concept for UltraClean and High-Efficiency Small DI Diesel Engines". SAE Technical Paper, 1999-01-3681, 10,

[4] Kook, S., Bae, C., Miles, P. C., Choi, D., and Pickett, L. M., 2005. "The influence of charge dilution and injection timing on low-temperature diesel combustion and emissions". SAE Technical Paper, 2005-01-3837, 10,.

[5] Leach, F., Davy, M., Weall, A., and Cooper, B., 2017. "Comparing the effect of a swirl flap and asymmetric inlet valve opening on a light duty diesel engine". In International Powertrains, Fuels Lubricants Meeting, SAE International.

[6] Papaioannou, N., Leach, F. C., Davy, M. H., Weall, A., and Cooper, B., 2019. "Evaluation of exhaust gas recirculation techniques on a high-speed direct injection diesel engine using first law analysis". Proceedings of the Institution of Mechanical Engineers, Part D: Journal of Automobile Engineering, 233(3), pp. 710-726.

[7] Mendez, S., and Thirouard, B., 2008. Using multiple injection strategies in diesel combustion: Potential to improve emissions, noise and fuel economy trade-off in low cr engines, apr.

[8] Mingfa, Y., Hu, W., Zunqing, Z., and Yan, Y., 2009. "Experimental study of multiple injections and coupling effects of multi-injection and egr in a hd diesel engine". In SAE 2009 Powertrains Fuels and Lubricants Meeting, SAE International.

[9] Schiffmann, P., Reuss, D. L., and Sick, V., 2018. "Empirical investigation of spark-ignited flame-initiation cycleto-cycle variability in a homogeneous charge reciprocating engine". International Journal of Engine Research, 19(5), pp. 491-508.

[10] Alcan, G., Unel, M., Aran, V., Yilmaz, M., Gurel, C., and Koprubasi, K., 2018. "Diesel engine nox emission modeling using a new experiment design and reduced set of regressors". IFAC-PapersOnLine, 51(15), pp. 168 - 173. 18th IFAC Symposium on System Identification SYSID 2018.

[11] Desantes, J., Lopez, J., Garcia-Oliver, J., and Hernández, L., 2002. "Application of neural networks for prediction and optimization of exhaust emissions in a h.d. diesel engine".

[12] Mohammadhassani, J., Khalilarya, S., Solimanpur, M., and Dadvand, A., 2012. "Prediction of nox emissions from a direct injection diesel engine using artificial neural network". Modelling and Simulation in Engineering, 2012, 05.
[13] Roy, S., Banerjee, R., and Bose, P. K., 2014. "Performance and exhaust emissions prediction of a crdi assisted single cylinder diesel engine coupled with egr using artificial neural network". Applied Energy, 119, pp. $330-340$.

[14] Badra, J., Khaled, F., Tang, M., Pei, Y., Kodavasal, J., Pal, P., Owoyele, O., Futterer, C., Brenner, M., and Farooq, A., 2019. "Engine combustion system optimization using $\mathrm{cfd}$ and machine learning: A methodological approach".

[15] , 2019. A Novel Active Optimization Approach for Rapid and Efficient Design Space Exploration Using Ensemble Machine Learning, Vol. ASME 2019 Internal Combustion Engine Division Fall Technical Conference of Internal Combustion Engine Division Fall Technical Conference. V001T06A006.

[16] Moiz, A. A., Pal, P., Probst, D., Pei, Y., Zhang, Y., Som, S., and Kodavasal, J., 2018. A machine learning-genetic algorithm (ml-ga) approach for rapid optimization using highperformance computing, apr.

[17] Badra, J., khaled, F., Sim, J., Pei, Y., Viollet, Y., Pal, P., Futterer, C., Brenner, M., Som, S., Farooq, A., and Chang, J., 2020. "Combustion system optimization of a light-duty gci engine using cfd and machine learning". In WCX SAE World Congress Experience, SAE International.

[18] Harris, S., Elliott, L., Ingham, D., Pourkashanian, M., and Wilson, C., 2000. "Optimization of reaction rate parameters for chemical kinetic modelling of combustion using genetic algorithms". Computer Methods in Applied Mechanics and Engineering, 190, 11, pp. 1065-1090.

[19] Li, W., and Chang, X., 2000. "Application of hybrid fuzzy logic proportional plus conventional integralderivative controller to combustion control of stoker-fired boilers”. Fuzzy Sets and Systems, 111(2), pp. 267 - 284.

[20] Parlak, A., Islamoglu, Y., Yasar, H., and Egrisogut, A., 2006. "Application of artificial neural network to predict specific fuel consumption and exhaust temperature for a diesel engine". Applied Thermal Engineering, 26(8), pp. $824-828$.

[21] Mauro, A. D., Chen, H., and Sick, V., 2019. "Neural network prediction of cycle-to-cycle power variability in a spark-ignited internal combustion engine". Proceedings of the Combustion Institute, 37(4), pp. 4937 - 4944.

[22] Hafner, M., Schaeler, M., and Isermann, R., 1999. "Fast neural networks for diesel engine control design". IFAC Proceedings Volumes, 32(2), pp. 8154 - 8159. 14th IFAC World Congress 1999, Beijing, Chia, 5-9 July.

[23] Isermann, R., and Hafner, M., 2001. "Mechatronic combustion engines - from modeling to optinlal control". European Journal of Control, 7(2), pp. $220-247$.

[24] X. Fang, N. Papaioannou, F. L. M. D., 2020. “On the application of artificial neural networks for the prediction of no $x$ emissions from a high-speed direct injection diesel engine”. International Journal of Engine Research, Accepted. 
[25] Leach, F., Ismail, R., Davy, M., Weall, A., and Cooper, B., 2018. "The effect of a stepped lip piston design on performance and emissions from a high-speed diesel engine". Applied Energy, 215, pp. $679-689$.

[26] Papaioannou, N., Leach, F., and Davy, M., 2019. "Thermal analysis of steel and aluminium pistons for an hsdi diesel engine". In WCX SAE World Congress Experience, SAE International.

[27] Papaioannou, N., Leach, F., Davy, M., and Gilchrist, R., 2020. "The effect of an active thermal coating on efficiency and emissions from a high speed direct injection diesel engine". In WCX SAE World Congress Experience, SAE International.

[28] Gabriel, D., and Hettich, T., 2015. "Topweld $\AA$ steel piston for high speed diesel engines". In SAE 2015 World Congress Exhibition, SAE International.

[29] Ladommatos, N., Xiao, Z., and Zhao, H., 2005. "The effect of piston bowl temperature on diesel exhaust emissions". Proceedings of the Institution of Mechanical Engineers, Part D: Journal of Automobile Engineering, 219(3), pp. 371-388.

[30] Schreer, K., Roth, I., Schneider, S., and Ehnis, H., 2014. "Analysis of Aluminum and Steel Pistons-Comparison of Friction, Piston Temperature, and Combustion". Journal of Engineering for Gas Turbines and Power, 136(10), 05. 101506. 